\section{OBLICON: A FORTRAN IV program for oblique confactor rotation}

\author{
DAVID JOHN GOW \\ Department of Political Science \\ Rice University, Houston, Texas 77001
}

It is well known that in factor analysis the matrix of factor loadings may, without loss, be transformed in a variety of ways. This fundamental indeterminacy in the factor model has led to a plethora of techniques (such as varimax) to transform factor matrices to simple structure. When an hypothesis has been advanced about the structure of the factor loadings, then confirmatory factor analysis (Jöreskog, 1969) or a variety of procrustes procedures (Hurley \& Cattell, 1962; Evans, 1971; Schönemann, 1966) may be used.

Confactor rotation, on the other hand, requires no particular hypothesis about the loadings, but rather two (or more) explicitly designed parallel experiments utilizing the same variables. Different sample or stimulus conditions will generally result in comparable factors having different variances in the two experiments. Cattell and Brennan (1977) argue that "real influences will appear as factors but that only one of the infinite possible series of rotated factors will correspond with the real influences. If a real influence exists and is more strongly at work in one experiment than the other, then at the right position for both experiments the profile of loadings should be the same as that in the other multiplied throughout by a ratio $d "$ "(p. 95).

Cattell and Cattell (1955) solved the problem of orthogonal confactor rotation. Cattell and Brennan (1977) extended the work to investigate the circumstances under which the orthogonal model provides good approximations of the oblique case. The importance of oblique rotation is that it allows the investigation of higher order source traits; consequently, oblique factors play a very important role in factorbased personality theory. No solution has previously been proposed for the oblique confactor model. The probable reason is that with fallible data no algebraic solution is possible. However, Gow (Note 1) has proposed an iterative technique that solves the oblique confactor model. This procedure will generally converge to the required least-squares solution. OBLICON implements the algorithm proposed by Gow for oblique confactor rotation.

The oblique confactor model may be stated in the following terms. Given two matrices, $A$ and B, both of order $n$ by $m$ (where $n>m$ ), determine two transformation matrices, $T$ and $U$, both of order $m$ by $m$, and a $\mathrm{m}$ by $\mathrm{m}$ diagonal matrix, $\mathrm{D}$, such that:

$$
\mathrm{AT}=\mathrm{BUD}+\mathrm{E}
$$

under the constraints that

$$
\operatorname{diag}\left(T^{\prime} T\right)=\operatorname{diag}\left(U^{\prime} U\right)=\operatorname{diag}(I)
$$

The matrix $\mathrm{E}$ is a matrix of residuals. The function to be minimized is the trace of $E^{\prime} E$ under the constraints of Equation 2. It may be noted that, when $\mathrm{T}$ and $\mathrm{D}$ are identity matrices, the oblique confactor model contains the oblique procrustes model (Browne, 1967) as a special case. The OBLICON program may be used for this and other special cases.

In order to minimize the function, an iterative process is used. This entails using an alternating leastsquares (de Leeuw, Young, \& Takane, 1976) or zig-zag (Oberhofer \& Kmenta, 1974) approach. The function is minimized conditionally for each matrix, given estimates of the other matrices. That is, the function is minimized conditionally for $\mathrm{U}$, given some estimate of $\mathrm{T}$ and $\mathrm{D}$, then minimized conditionally for $\mathrm{T}$, given $\mathrm{D}$ and U. Finally, the conditional least-squares estimate for $\mathrm{D}$ is obtained given $\mathrm{T}$ and $\mathrm{U}$. This completes one iteration. In each of the three miniproblems, the estimates that are obtained have least-squares properties. Consequently, the trace of $E^{\prime} E$ should be nonincreasing over successive iterations.

Input. OBLICON requires as input the two matrices $\mathrm{A}$ and $\mathrm{B}$, both of which are of order $\mathrm{n}$ by $\mathrm{m}$. Further input consists of a problem parameter card that allows the user to control various aspects of the iterative process, including solutions for various submodels of the oblique confactor model stated in Equation 1. Typically, A and B are matrices of factor loadings from two experiments or samples utilizing the same variables.

Output. The output from OBLICON consists of a detailed history of the iterative process together with the final solution for the transformation matrices, $\mathrm{U}, \mathrm{D}$, and $\mathrm{T}$. After each iteration the current estimates of the matrices, T, U, D, E, and AT, BU and BUD are printed together with the evaluation of the function. The final solution, after convergence of the iterations, also reports the direction cosines among the transformation matrices.

Limitations. The present version of OBLICON is dimensioned to handle matrices $A$ and $B$, both of which may be of orders up to 99 by 9 . Typically, this consists of 99 variables and 9 factors. The program requires $150 \mathrm{~K}$ bytes; consequently, larger problems can be accommodated if further memory is available. Medium-size problems (where $\mathbf{A}$ and $\mathbf{B}$ are of order 20 by 6) typically use less than $20 \mathrm{sec}$ of CPU time on an IBM $370 / 155$. Consequently, the program is reasonably rapid, and the most meaningful limitation of the application of OBLICON is available memory.

Computer and Language. OBLICON may be compiled under FORTRAN IV(G) on an IBM 370/155. The 
program is coded in such a way that it can easily be implemented on other compilers. Due to the iterative nature of the algorithm, double precision is used in all noninteger computations. Internal comment statements clearly document potential problem areas in conversion to non-IBM compilers.

Availability. The source deck for OBLICON is available from David John Gow, Department of Political Science, Rice University, Houston, Texas 77001. A program write-up is also available which provides detailed instructions about the use and implementation of the program. The source deck and the write-up are free of charge.

\section{REFERENCE NOTE}

1. Gow, D. J. An alternating least-squares approach to the resolution of oblique factors in confactor rotation. Submitted for publication, 1978.

\section{REFERENCES}

Browne. M. W. On oblique procrustes rotation. Psychometrika, $1967,32,125-132$.

Cattell. R. B. "Parallel proportional profiles" and other principles for determining the choice of factors by rotation. Psychometrika. 1944, 9, 267-283.
Cattell. R. B.. \& Brennan, J. The practicality of an orthogonal confactor rotation for the approximate resolution of oblique factors. Multivariate Experimental Clinical Research, 1977, 3, 95-104.

Cattell, R. B., \& Cattell, A. K. S. Factor rotation for proportional profiles: Analytical solution and an example. British Journal of Mathematical and Statistical Psychology, 1955, 8, 83-92.

De Leeuw, J., Young, F. W., \& Takane, Y. Additive structure in qualitative data: An alternating least squares method with optimal scaling features. Psychometrika, 1976, 41, 471-503.

Evans, G. T. Transformation of factor matrices to achieve congruence. British Journal of Mathematical and Statistical Psychology, 1971, 24, 22-48.

Hurley, J. R., \& Cattell, R. B. The procrustes program: Producing direct rotation to test a hypothesized factor structure. Behavioral Science, 1962, 7, 258-262.

JöRESKOG, K. G. A general approach to confirmatory maximumlikelihood factor analysis. Psychometrika, 1969, 34, 183-202.

Oberhofer, W., \& Kmenta, J. A general procedure for obtaining maximum likelihood estimates in generalized regression models. Econometrica, 1974, 42, 579-590.

SChönemanN, P. H. A generalized solution of the orthogonal procrustes problem. Psychometrika, 1966, 31, 1-10.

(Accepted for publication January 4, 1978.) 